\title{
Processing of Emotion Words by Patients with Autism Spectrum Disorders: Evidence from Reaction Times and EEG
}

\author{
Alina Lartseva · Ton Dijkstra • Cornelis C. Kan • \\ Jan K. Buitelaar
}

Published online: 12 June 2014

(c) Springer Science+Business Media New York 2014

\begin{abstract}
This study investigated processing of emotion words in autism spectrum disorders (ASD) using reaction times and event-related potentials (ERP). Adults with $(\mathrm{n}=21)$ and without $(\mathrm{n}=20)$ ASD performed a lexical decision task on emotion and neutral words while their brain activity was recorded. Both groups showed faster responses to emotion words compared to neutral, suggesting intact early processing of emotion in ASD. In the ERPs, the control group showed a typical late positive component (LPC) at $400-600 \mathrm{~ms}$ for emotion words compared to neutral, while the ASD group showed no LPC. The between-group difference in LPC amplitude was
\end{abstract}

A. Lartseva · J. K. Buitelaar

Department of Cognitive Neuroscience, Donders Institute for Brain, Cognition and Behaviour, Radboud University Nijmegen Medical Centre, Geert Grooteplein Noord 21,

6525 EZ Nijmegen, The Netherlands

\section{A. Lartseva}

International Max Planck Research School for Language

Sciences, Wundtlaan 1, 6525 XD Nijmegen, The Netherlands

A. Lartseva $(\square)$

Kapittelweg 29, 6525 Nijmegen, The Netherlands

e-mail: a.lartseva@gmail.com; a.lartseva@donders.ru.nl

T. Dijkstra

Donders Institute for Brain, Cognition and Behaviour, Radboud University Nijmegen, Montessorilaan 3, 6525 HR Nijmegen,

The Netherlands

C. C. Kan

Department of Psychiatry, Radboud University Nijmegen

Medical Centre, Reinier Postlaan 4, 6525 GC Nijmegen,

The Netherlands

J. K. Buitelaar

Karakter Child and Adolescent Psychiatry University Centre,

Nijmegen, The Netherlands significant, suggesting that emotion words were processed differently by individuals with ASD, although their behavioral performance was similar to that of typical individuals.

Keywords Autism spectrum disorders - EEG - ERP . Emotion words $\cdot$ LPC $\cdot$ Lexical decision task

\section{Introduction}

Autism spectrum disorders (ASD) are characterized by problems in reciprocal social interaction, verbal and nonverbal communication, as well as by rigid and stereotypical behavioral patterns (APA 2000). At present, a precise understanding of emotion processing in ASD is lacking. We used reaction time measures to investigate whether adult individuals with ASD process emotion words differently from non-ASD controls, and we obtained EEG measures to examine whether any observed differences occur at an early or late stage of processing.

Most studies of emotion processing in ASD focused on facial expressions of emotion (Harms et al. 2010; Jemel et al. 2006). However, in recent years, the processing of emotion in non-facial stimuli in ASD became an increasingly important topic. The traditional position is that emotional impairments are foremost linked to and due to the well-known social deficits in ASD. Many researchers also linked problems in emotion understanding to Theory of Mind deficits (Baron-Cohen et al. 2001; Heerey et al. 2003; Hillier and Allinson 2002). In contrast, other scholars argued that emotional impairments are more widespread and extend beyond the social domain (e.g., Gaigg 2012). Yet other authors argue that emotional impairments are not universal in ASD, which means that there is a lot of 
heterogeneity in the results across different groups of ASD participants and across different tasks, and that the impairments are not specific to emotion (Nuske et al. 2013). This implies that many of the findings can be accounted for in terms of differences in attention/motivation/baseline physiological activity between ASD and comparison groups.

For typical participants, the processing of emotion words and images has been well-studied. First of all, both emotionally positive and negative words are more salient and automatically recruit attentional resources: they are remembered better than neutral words (Kensinger and Corkin 2003), and when participants are asked to perform a lexical decision task (in which they decide as quickly as possible whether a letter string is an existing word or not), they respond faster (Kousta et al. 2009; Kuchinke et al. 2007), and more accurately (Eviatar and Zaidel 1991) if the word is emotional. However, a difference arises not only between emotional and neutral words, but also between emotionally positive and emotionally negative words. For example, emotionally negative words produce interference effects in the color naming Stroop task, but this effect has not been obtained with emotionally positive words (Mackay et al. 2004). In a detection task, emotionally negative words are generally detected faster and more accurately than positive words (Nasrallah et al. 2009), but in a classification task people take longer to classify emotionally negative words (Dahl 2001). In the lexical decision task, the effect for negative words is generally weaker (Kissler and Koessler 2011) and negative words with lower emotional arousal ratings are in fact recognized slower, while positive words are recognized faster irrespective of arousal (Hofmann et al. 2009). One possible explanation for this response difference to positive and negative emotion words is that positive emotion additionally triggers an approach tendency and facilitates responses, while negative emotion triggers avoidance, thus causing a slowdown. In other words, for positive emotion, the two effects are in line and add up, but for negative words they work in opposite direction; the observed outcome depends on which effect dominates in this specific task (Kousta et al. 2009). Another possibility is that emotionally negative words recruit more cognitive resources, thus interfering with the task (Dahl 2001).

Event-related potential (ERP) studies show that the earliest effects of emotion are visible as early as $100 \mathrm{~ms}$ after word onset (Hofmann et al. 2009). Two ERP correlates of emotion processing are the early posterior negativity (EPN) and the late positive component (LPC).

The EPN peaks between 250 and $300 \mathrm{~ms}$ with a posterior distribution; it is associated with early detection of emotionally salient stimuli (Schacht and Sommer 2009; Kissler et al. 2009; Herbert et al. 2008). However, some studies do not find an EPN effect (Hofmann et al. 2009; Dillon et al. 2006), and other studies report an enhanced P200/P300 amplitude for emotional stimuli, an effect in the opposite direction compared to EPN (Herbert et al. 2006; Kanske and Kotz 2007).

The LPC typically starts around $400 \mathrm{~ms}$ after stimulus onset, and lasts for several hundred milliseconds (Dillon et al. 2006; Schacht and Sommer 2009). Some studies observe similar LPC effects for positive and negative words (Schacht), while other studies find bigger LPC for positive words (Herbert 2006), and yet other studies find a more negative ERP for emotionally negative words (Herbert 2008). A similar LPC effect has been found not only for single words, but also for emotion words embedded in sentence context (Bayer et al. 2010; Holt et al. 2008) and for emotional images (Schupp et al. 2000). The LPC has been associated with motivational engagement (Schupp et al. 2000), enhanced attention, and deeper stimulus encoding (Herbert et al. 2006). For example, a study with images found that increased LPC amplitudes for emotional items correlated with better memory performance on those items (Dolcos and Cabeza 2002).

To date, only a few studies have investigated emotional processing in ASD outside the domain of facial emotion recognition. Studies on memory performance found that emotional valence had little or no effect in the ASD group, in contrast to the typical population when participants were asked to remember and subsequently recall emotional sentences (Beversdorf et al. 1998), images (Wilbarger et al. 2009; Deruelle et al. 2008), and single words (Gaigg and Bowler 2008, 2009b). However, other studies failed to replicate this finding (South et al. 2008). In a sequence of rapidly presented stimuli, typical participants detected emotional words more accurately than neutral words, but this was not the case in the ASD group (Corden et al. 2008; Gaigg and Bowler 2009a). Finally, two more studies reported that individuals with ASD display an abnormal pattern of automatic reflexes such as startle reflex and postauricular reflex in response to emotional stimuli (Dichter et al. 2010; Wilbarger et al. 2009). It is not clear whether there are differential effects of negative valence in ASD group. Several of the studies mentioned above only used negative emotional stimuli (Beversdorf et al. 1998; Corden et al. 2008). One study using emotionally positive and negative items found an effect for negative, but not for positive emotion (Deruelle et al. 2008).

In the current study, we investigated for the first time the emotion facilitation effect in the ASD population in a lexical decision task. In the comparison group with typical participants, we expected to find the often observed emotion facilitation effect (cf Kousta et al. 2009) and the LPC component for emotion words in the EEG. In line with previous research, we expected that in the ASD sample, 
word valence would have little or no effect on reaction times and ERP amplitudes.

Failure to find ERP effects of emotion in the ASD group could be due to other reasons than just issues of valence. For example, it could be that participants in our sample do not show a reliable ERP response, or that they have a general impairment in lexical-semantic processing which is not specific to emotion per se, but affects all aspects of word processing. Response times and ERP amplitudes to words in general are not only influenced by emotional valence, but also by word frequency (Grainger 1990; Rugg 1990; Hauk and Pulvermüller 2004; Holcomb and Grainger 2006). Specifically, word frequency modulates the N400 ERP component in the time window between 300 and $500 \mathrm{~ms}$ (Halgren et al. 2002; Hauk and Pulvermüller 2004), with low-frequency words eliciting more negative amplitudes compared to high-frequency words. The use of the word frequency as an additional control variable provides a safeguard that any absence of a valence effect is not due to an insensitivity of the experimental procedure. Absence of the effect of frequency in addition to an effect of valence would point to a more general impairment in language processing, while the presence of frequency effects in the absence of valence effects would indicate that findings are really valence-specific.

\section{Materials and Methods}

\section{Participants}

Participants included 21 high-functioning adults with ASD and 20 matched typical individuals (15 males), aged 18-36 years. All participants with ASD met the DSM-IV (DSM-IV-TR, 2000) criteria for autistic disorder or Asperger syndrome as established by an independent clinician. The clinical diagnosis was established based on all information collected during a psychiatric interview, developmental history, an interview with the parents, if available, and a review of prior clinical records. In ten subjects, the Autism Diagnostic Interview-Revised (ADIR) (Lord et al. 1994) could be administered, the results of which confirmed the clinical diagnosis. In all cases, the clinical diagnosis of ASD was beyond doubt. People with a PDD-NOS diagnosis or severe comorbid axis-I conditions (schizophrenia, bipolar disorder, or depression) were excluded.

The ASD group was recruited from referrals to the Department of Psychiatry at the UMC and from participants from previous studies (Groen et al. 2009; Poljac et al. 2009; Visser et al. 2013). People from the comparison group were recruited through advertisements in the local community. Prior to inclusion, typical subjects were asked whether they had any history of psychiatric or neurological
Table 1 Participant characteristics

\begin{tabular}{llll}
\hline & $\begin{array}{l}\text { Typical } \\
(\mathrm{n}=20)\end{array}$ & $\begin{array}{l}\text { ASD EEG } \\
\text { sample } \\
(\mathrm{n}=19)\end{array}$ & $\begin{array}{l}\text { ASD } \\
\text { behavioural } \\
\text { sample } \\
(/=21)\end{array}$ \\
\hline Females/males & $5 / 15$ & $5 / 14$ & $7 / 14$ \\
Age & $24.3(4.3)$ & $26.7(5.8)$ & $26.9(5.6)$ \\
Verbal IQ (PPVT) & $103.8(9.3)$ & $102.7(14.2)$ & $103.6(13.9)$ \\
Raven (raw score) & $23.1(7.7)$ & $24.4(7.8)$ & $24.5(7.4)$ \\
Raven (IQ score) & $107.9(15.8)$ & $108.6(14.4)$ & $108.8(13.7)$ \\
\hline
\end{tabular}

disorders; subjects diagnosed with any of these disorders were excluded. All participants were native speakers of Dutch and had no known history of neurological disorder, head injury or reading problems. All participants had normal or corrected-to-normal vision. Two participants with ASD had used psychostimulant medication due to comorbid ADHD prior to the current experiment. All participants gave informed consent to participate in the study and were reimbursed for participation at the rate of 8 euros per hour and travel expenses. The study was formally approved by the local medical ethics committee.

IQ was assessed with the adult version of the Peabody Picture Vocabulary test (Manschot and Bonnema 1974) and Raven Progressive Matrices test (Raven et al. 1998) in all participants. The groups did not significantly differ on age, verbal intelligence, and nonverbal intelligence $(p>.1$ for all variables).

To control for possible differences in mood, participants were asked to fill out the profile of mood states (POMS) questionnaire (Wald and Mellenbergh 1990) immediately after the experiment. The participant characteristics are summarized in Table 1.

\section{Materials}

The stimulus set consisted of 180 Dutch words and 180 pseudowords. Words consisted of 60 neutral, 60 positive, and 60 negative Dutch nouns. Before the study, we collected ratings on their valence (emotionally positive or negative), arousal (relaxing or arousing), and concreteness (concrete or abstract) by means of an online survey. Each word was rated by at least 25 people who did not participate in the main experiment. All ratings were collected on a 7-point Likert scale.

Positive and negative words were matched on perceived arousal ability, and differed significantly from neutral words with respect to both valence and arousal. All three word groups were matched on concreteness, frequency collected from the CELEX database (at http://celex.mpi.nl, (Baayen et al. 1995), and length. The nonwords consisted of legitimate Dutch letter combinations. They were 
Table 2 Stimuli characteristics

\begin{tabular}{llll}
\hline & Positive & Neutral & Negative \\
\hline High-frequency & & & \\
Valence $^{\mathrm{a}}$ & 5.76 & 4.08 & 1.9 \\
Arousal $^{\mathrm{a}}$ & 5.2 & 3.25 & 5.04 \\
Concreteness $^{\mathrm{a}}$ & 4.69 & 4.48 & 4.56 \\
Frequency $^{\mathrm{a}}$ & 55.9 & 56.7 & 55.9 \\
Length & 6.37 & 6.17 & 6.27 \\
Low-frequency & & & \\
Valence $^{\mathrm{a}}$ & 5.72 & 4.02 & 1.87 \\
Arousal $^{\mathrm{a}}$ & 5.1 & 3.15 & 4.96 \\
Concreteness $^{\mathrm{a}}$ & 4.81 & 4.63 & 4.76 \\
Frequency & 8.5 & 8.7 & 8.4 \\
Length & 6.47 & 6.53 & 6.6 \\
\hline
\end{tabular}

a Rated on a 1-7 scale

b Per million tokens in the CELEX text corpus

matched to the words with respect to length in number of letters.

Additionally, we controlled the stimuli on lexical frequency per million [number of occurrences of a word in the CELEX text corpus per million words (Baayen et al. 1995)]. To investigate the effect of frequency, we divided the stimuli into a high-frequency and a low-frequency condition, making sure that frequency and emotion were manipulated orthogonally. Table 2 summarizes the word characteristics: ratings of valence, arousal and concreteness, length in letters, and CELEX frequency per million.

\section{Procedure}

Participants were seated in a dimly lit, sound-attenuated room in front of a computer screen. The monitor was approximately $60 \mathrm{~cm}$ away, and the participants were allowed to adjust it to a comfortable distance. All stimuli were presented in white capital letters in Arial font against dark gray background at the center of the screen. Each trial began with a fixation cross for $300 \mathrm{~ms}$, after which a letter string was presented for $350 \mathrm{~ms}$, followed by a blank screen. The participants were instructed to read the letter string and respond whether it was an existing Dutch word or not by pressing a "yes" or "no" button on a buttonbox. The response interval lasted for maximally $2,000 \mathrm{~ms}$ and ended with a button press. The intertrial interval between response/timeout and the next trial lasted for 2,000 ms.

\section{EEG Data Recording}

The EEG was recorded from 64 ActiCap active electrodes (Brain Products GMBH). The signal was amplified with two BrainAmp EEG amplifiers, powered by BrainVision
Powerpack LiOn rechargeable batteries. Sixty test and reference electrodes were placed in the Easycap EEG recording cap in an equidistant montage (M10 Equidistant 61-Channel-Arrangement), and four electrodes were placed above and below left eye and at the outer side of each eye to record eye movement. The ground was placed at the nasion and the reference was placed at the left mastoid. The impedance was kept below $20 \mathrm{kOhm}$. We used the Brain Vision Recorder Professional software (Brain Products $\mathrm{GmbH}$ ) for the recording. The signal was sampled at $500 \mathrm{~Hz}$, and then band-pass amplified with upper limit at $200 \mathrm{~Hz}$, using a time constant of $10 \mathrm{~s}$.

\section{Data Analysis}

\section{Behavioral Data Analysis}

The error rate was below $10 \%$ in all participants, with the exception of one participant who produced an error rate of $18 \%$ ( $3 \%$ of responses were false rejections of word trials and $15 \%$ were false "yes" responses to nonwords). Removing the data of this participant did not change the pattern of results; therefore in the final analysis we report the results from all participants. For 5 words out of 120, more than 10 participants $(25 \%)$ gave an incorrect response. These words were excluded from further analysis. Additionally, we excluded all trials with very slow or very fast responses. The cutoff point for slow responses was set at 2.5 standard deviations of the subject mean (this is a common cutoff threshold in psycholinguistic studies, see for example Yap et al. 2013; Chwilla et al. 2000; van Hell and Dijkstra 2002), and was calculated for every participant separately. The cutoff point for the fast responses was set at $100 \mathrm{~ms}$ after stimulus onset. The preprocessing was done using Matlab R2009b (The MathWorks, Inc., Natic, MA) software.

To make sure that the results were not driven by the slow responses, we repeated the same analysis for the nonpreprocessed data (with only nonwords and false responses removed). We found the same pattern of results as in the preprocessed data.

As an estimate of effect size in repeated-measures ANOVA we report the generalized eta squared $\left(\eta_{G}^{2}\right)$ in addition to partial eta squared $\left(\eta_{\mathrm{p}}^{2}\right)$. The first measure is proposed as a preferred effect size measure for withinsubject designs, which provides comparability for withinand between-subjects designs (Bakeman 2005).

EEG Data Analysis: Whole Surface

Because of technical problems during registration, the EEG data from two participants with ASD could not be 
analyzed. The EEG data were analyzed with Fieldtrip software (Oostenveld et al. 2011). The segments were defined from $200 \mathrm{~ms}$ before word onset until 1,000 ms after word onset. Trials with muscle artifacts were detected based on power in $110-140 \mathrm{~Hz}$ frequency band and rejected completely. Eyeblink artifacts were removed with the Independent Component Analysis (ICA) method implemented in Fieldtrip. After that, the data were lowpass filtered at $35 \mathrm{~Hz}$, preprocessed to remove the linear trend, baseline corrected using a $200 \mathrm{~ms}$ interval before word onset as a baseline, and then converted to an average reference. Next, all trials were manually reviewed and remaining artifacts were removed. Finally, the data were distinguished into different conditions and a grand average was calculated for each condition separately.

For the EEG data analysis, we used permutation-based statistics. A conventional ERP analysis is based on a specific time interval and location of interest selection. However, the selection has to be done prior to the analysis, otherwise it leads to biased effect reporting and inflated statistical significance, which is a serious concern (Kilner 2013). For typical individuals, previous studies allow us to make a definite prediction about where and when the effect of interest will appear. However, for the ASD group no such studies have been done, and if the effect appears earlier or later or has a different spatial distribution, we might entirely miss it. Permutation-based statistics based on a whole-surface whole-time interval analysis avoids this problem by looking at the entire data set while correcting for multiple comparisons.

Instead of averaging the data over a given time interval and channel group, we calculated a $t$ test for every channel and every time point, and looked for clusters of data points exceeding an uncorrected significance threshold of $p=.05$. The cluster statistic was defined as the sum of individual $\mathrm{t}$-values. The significance threshold for the cluster statistic was determined using Monte Carlo simulations by randomly permuting the original data and calculating the probability distribution for the cluster statistic over 1,000 simulations. The cluster statistic was considered as significant if its value was exceeded in no more than $5 \%$ cases in the permuted data. This method was developed specifically for EEG/MEG data, and it is similar to a Monte Carlo simulation-based approach used for fMRI data analysis. (For further details and the application of this method to EEG/MEG data, see (Maris and Oostenveld 2007; van Ede et al. 2011; Moratti et al. 2011).

\section{EEG Data Analysis: Regions of Interest}

In addition to the whole brain analysis, we also ran a region of interest (ROI) analysis. We analyzed the data by region and time of interest as follows. From the literature we

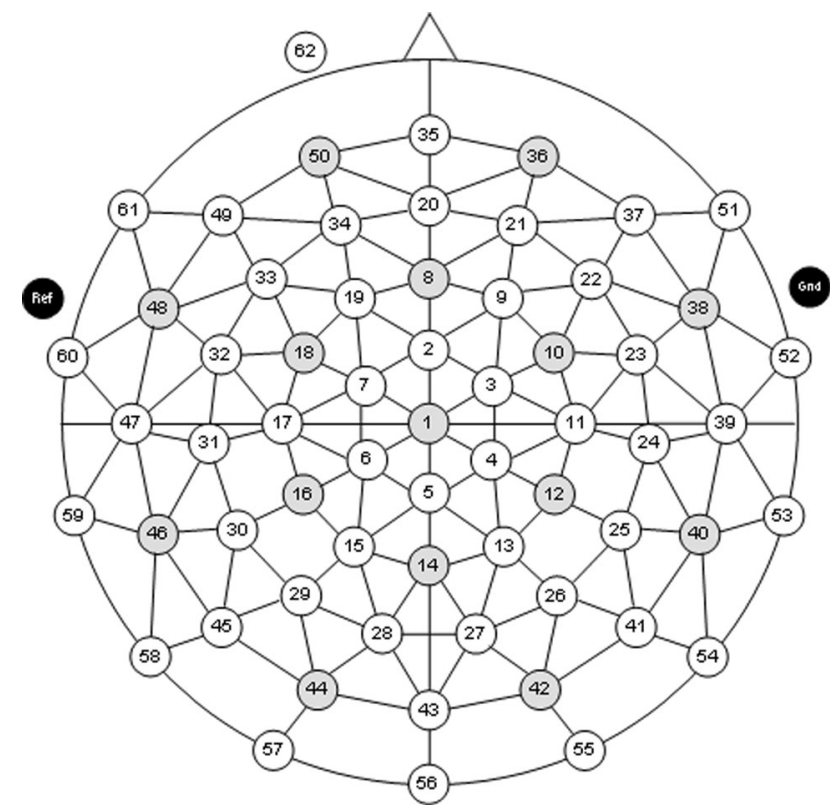

Fig. 1 Electrode positions in the M10 equidistant montage

know that the effect of lexical frequency is most commonly found in the N400 time window (between 300 and $500 \mathrm{~ms}$ ), and the effect of emotion (LPC) is present between 400 and $900 \mathrm{~ms}$ with some variation between the studies. Therefore, we defined two time intervals of interest: the N400 window (300-500 ms) and the LPC time window (500-700 ms).

Previous studies have found that the effects of frequency and emotion show a centro-parietal distribution. Therefore, a ROI midline analysis would be most adequate to investigate the effects. We defined three ROIs: the Fz group (electrodes 2,8,9,19,20), the $\mathrm{Cz}$ group (electrodes $1,3,4,6,7$ ), and the $\mathrm{Pz}$ group (electrodes $13,14,15,27,28$ ) (See Fig. 1).

We expect to find a significant effect of frequency in the N400 time window with no differences between group, and we plan to find an effect of valence in the N400 and the LPC time windows and a significant between-group difference.

\section{Results}

\section{Behavioral Results}

The repeated measures ANOVA with reaction time as dependent variable, and Group (ASD, typical) and Emotion (positive, neutral, negative) as independent variables yielded a significant main effect of group $(\mathrm{F}(1,39)=9.47$, $\left.p<.005, \eta_{\mathrm{p}}^{2}=.20 ; \eta_{\mathrm{G}}^{2}=.19\right)$ and a significant main effect of Emotion $\left(\mathrm{F}(2,78)=23.60, p<.001, \eta_{\mathrm{p}}^{2}=.38\right.$, 
Fig. 2 Mean reaction times for emotional and neutral words in the ASD and typical groups (error bars indicate $95 \%$ confidence intervals). The numbers indicate the error rate (in percent) for each condition, standard deviation is in parentheses

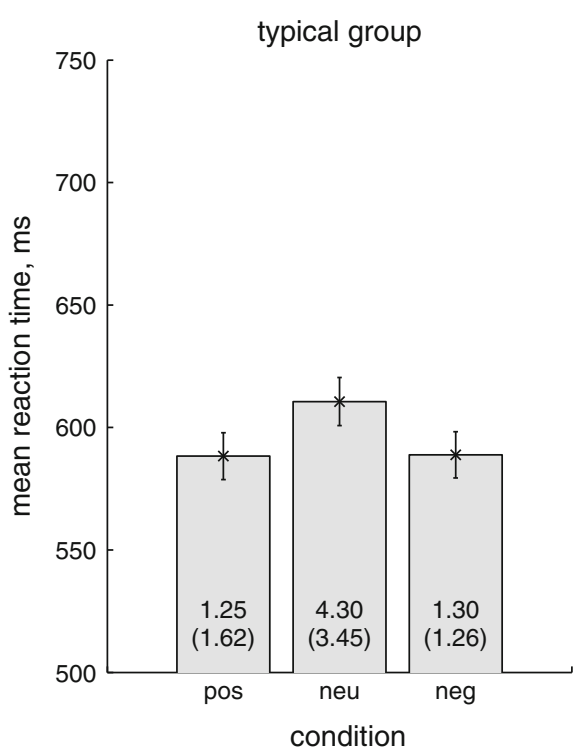

$\left.\eta_{\mathrm{G}}^{2}=.007\right)$. The typical subjects reacted overall faster than the ASD subjects, and reactions to emotion words were overall faster than reactions to neutral words (see Fig. 2). The interaction between the two factors was not significant $(\mathrm{F}=.29, p=.74)$.

Although the two groups did not statistically differ in terms of their IQ, we explored whether the group effect was co-determined by IQ. When verbal IQ measured by PPVT was added as a covariate to the model, the main effects of Group and Emotion remained significant, and the interaction between the two remained nonsignificant. However, the Emotion * Group * PPVT interaction was found to be significant (Greenhouse-Geisser corrected, F(4, 74) $\left.=2.98, p=.024, \eta_{p}^{2}=.14\right)$. This means that verbal IQ played a different role in the ASD and typical groups. In order to explore the relationship between verbal IQ and the emotion facilitation effect in ASD and typical individuals, we looked at the two groups separately. In the typical group, the emotion facilitation effect (which is the reaction time difference between emotion and neutral words) was not significantly correlated with the verbal IQ of the participants for either emotionally positive words $(r=-.23$, $p=.34)$, or emotionally negative words $(\mathrm{r}=-.03$, $p=.9$ ). In the ASD group, the verbal IQ of the participants was uncorrelated with the emotion facilitation effect for positive words $(\mathrm{r}=-.38, p=.09)$, but significantly correlated with the emotion facilitation effect for negative words $(\mathrm{r}=-.56, p=.008)$. ASD participants with higher verbal IQ score displayed a smaller reaction time difference between negative and neutral words.

In the analysis by items with between-subject factors Group (ASD, typical) and Emotion (positive, negative, neutral), both the factors Group $(\mathrm{F} 2(1,344)=543.0, p<.001$, $\left.\eta_{\mathrm{p}}^{2}=.61, \eta^{2}=.059\right)$ and Emotion $(\mathrm{F} 2(2,344)=10.88$, $p<.001, \eta_{\mathrm{p}}^{2}=.06, \eta^{2}=.02$ ) were significant. Adding the effects of frequency, concreteness, and length to the model did not change the significance level of the effect (main effect of Group: $\mathrm{F}(1,341)=628.83, p<.001$; main effect of Emotion: $\quad \mathrm{F}(2,341)=12.64, \quad p<.001 ; \quad$ frequency: $\mathrm{F}(1,341)=40.92, p<.001$; concreteness: $\mathrm{F}(1,341)=8.04$, $p=.005$; length: $\mathrm{F}(1,341)=4.40, p=.04$; for all interactions $p>$.2).

With respect to error rates in the analysis by subjects with factors Group (ASD, typical) and Emotion (positive, neutral, negative), there was no main effect of Group $(\mathrm{F}<.01, p=.93)$, but there was a main effect of Emotion, with emotional words eliciting more accurate responses compared to neutral words (Greenhouse-Geisser corrected, $\left.\mathrm{F}(2,78)=33.76, \quad p<.001, \quad \eta_{\mathrm{p}}^{2}=.46, \quad \eta_{\mathrm{G}}^{2}=.21\right) . \quad$ The interaction between group and word valence was not significant $(\mathrm{F}(2,78)=1.80, p=.18)$. The mean percentages of errors in the different conditions are reported in Fig. 2.

\section{EEG Results: Whole Surface Analysis}

In the analysis of the EEG data, we adopted the clusterbased statistics approach described above. Although at visual inspection the amplitude of the ERP components in the ASD group appeared to be smaller than in the typical group, between-group differences did not reach significance for any of the conditions (all $p$ values $>.1$ ).

Within-group comparisons between conditions yielded several significant results. First, a lexicality effect was found in both groups: nonwords elicited ERPs with more negative amplitudes compared to words. The timing and distribution of the lexicality effect was similar in the two groups (typical group: critical values for 2.5th and 97.5th percentile: $-1,377.1$ and 1,343.3 respectively, cluster 
(a)


Fig. 3 Lexicality and frequency effect in the ASD and typical group: a Average ERPs for the typical group for nonwords (black line), lowfrequency words (blue dashed line) and high-frequency words (blue

statistic $=19,404.2, p=.002,292-692 \mathrm{~ms}$; ASD group: critical values for 2.5 th and 97.5 th percentile: $-1,068.6$ and 995.9 respectively, cluster statistic $=9,113.2$, $p=.002,302-632 \mathrm{~ms})$. The group by lexicality interaction was not significant.

The effect of frequency was also found in both groups (see Fig. 3). High-frequency words elicited more positivegoing ERP than low-frequency words (typical group: critical values for 2.5 th and 97.5th percentiles: -781.2 and 720.8 respectively, cluster statistic $=5,568.9, p=.002$, time interval 394-564 ms; ASD group: critical values for 2.5th and 97.5th percentiles: -640.6 and 616.0 respectively, cluster statistic $=2,415.0, p=.002$, time interval 440-622 ms). Additionally, there was a marginally significant group by frequency interaction (critical values for 2.5th and 97.5th percentiles: -664.8 and 585.5 respectively, cluster statistic $=587.2, p=.05$, time interval 204-256 ms). In this time window, the typical group had higher voltage for high-frequency words compared to lowfrequency words, while in the ASD group this was reversed.

Turning to our main analyses, in the typical group both emotionally negative versus neutral conditions, and emotionally positive versus neutral conditions, significantly differed from each other. Emotionally positive words elicited a more positive-going ERP amplitude compared to neutral (critical values for 2.5th and 97.5th percentiles: -688.1 and 740.6 respectively, cluster statistic $=4,430.6$, $p=.002$, time interval $336-562 \mathrm{~ms}$ ); the same for emotionally negative versus neutral words (critical values for 2.5th and 97.5th percentiles: -812.5 and 940.8 respectively, cluster statistic $=5,082.7, p=.002$, time interval (b)

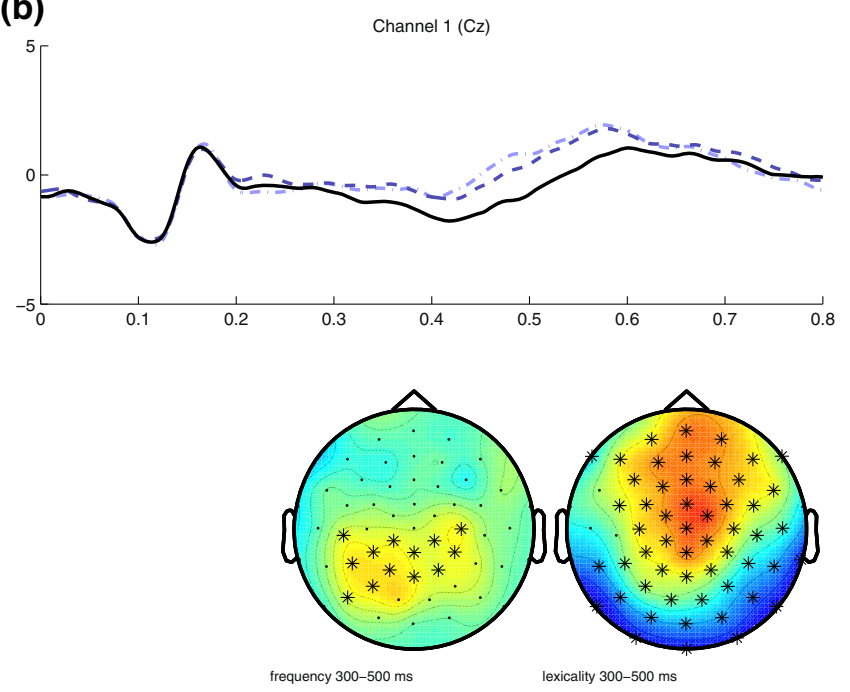

dash-dotted line). b Average ERPs for the ASD group for nonwords (black line), low-frequency words (blue dashed line) and highfrequency words (blue dash-dotted line) (Color figure online)

402-618 ms, see Fig. 4). The distribution of the significant clusters included left and central parietal electrodes. In the ASD group, however, the two conditions did not differ: positive versus neutral: critical values for 2.5 th and 97.5 th percentiles: -600.5 and 534.7 respectively, cluster statistic $=76.1, p=.9$, negative versus neutral: critical values for 2.5th and 97.5th percentiles: -568.6 and 500.5 respectively, cluster statistic $=295.8, p=.2$ (Fig. 4).

To test for between-group differences, we compared difference waves for positive versus neutral contrasts, and negative vs neutral contrasts, between groups. With respect to the difference between negative and neutral words, the two groups significantly differed from each other: the magnitude of the difference wave was larger in the typical group (critical values for 2.5th and 97.5th percentiles: -553.1 and 648.2 respectively, cluster statistic $=1,014.2$, $p=.014$, time interval $458-526 \mathrm{~ms}$ ). For the positive versus neutral contrast, the two groups did not differ statistically (critical values for 2.5 th and 97.5 th percentiles: -597.9 and 603.1 respectively, cluster statistic $=201.5$, $p=.5)$.

\section{EEG Results: Region of Interest Analysis}

To investigate the effect of frequency, we ran a Group (ASD, typical) by Frequency (high, low frequency) by Location $(\mathrm{Fz}, \mathrm{Cz}, \mathrm{Pz})$ repeated measures ANOVA with Group as between-subject factor, and Frequency and Location as within-subject factors within the N400 time window (300-500 ms). For the variables with more than three levels, the $p$ values were adjusted with GreenhouseGeisser correction. 

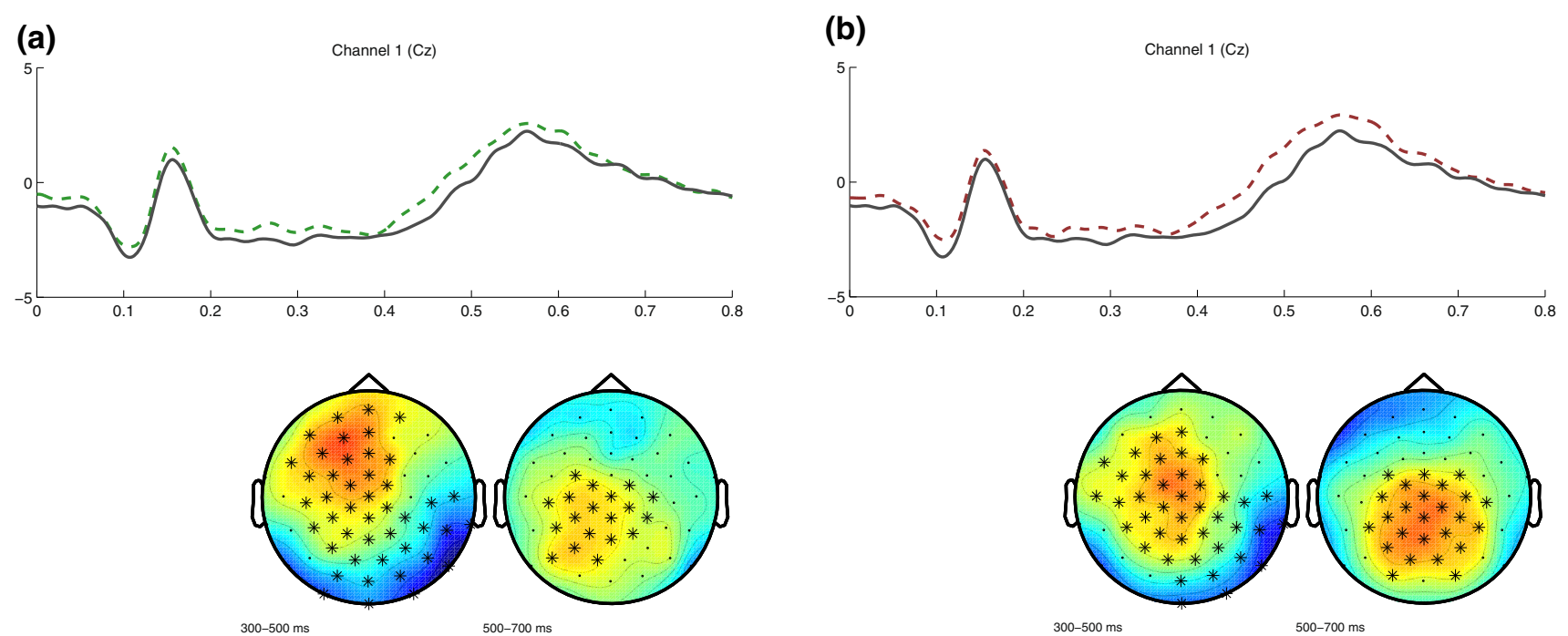

$300-500 \mathrm{~ms}$

$500-700 \mathrm{~ms}$

(c)
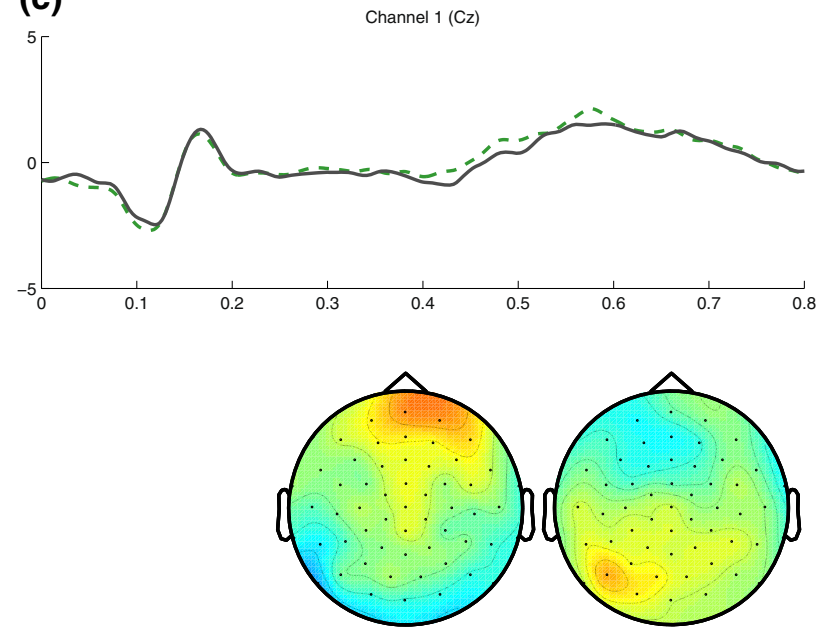

$300-500 \mathrm{~ms}$

$500-700 \mathrm{~ms}$

Fig. 4 Valence effect in ASD and typical group (asterisks indicate channels showing a significant difference between conditions): a typical group, positive words (green dashed line) versus neutral words (gray line), b typical group, negative words (red dashed line)

The ANOVA yielded a significant main effect of Frequency $\left(\mathrm{F}(1,37)=7.7, p=.009, \eta_{\mathrm{p}}^{2}=.17, \eta_{\mathrm{G}}^{2}=.003\right)$, main effect of Location $\left(\mathrm{F}(2,74)=28.1, p<.001, \eta_{\mathrm{p}}^{2}=.43\right.$, $\left.\eta_{\mathrm{G}}^{2}=.34\right)$ and a significant Location by Frequency interaction $\left(\mathrm{F}(2,74)=7.32, p=.004, \eta_{\mathrm{p}}^{2}=.172, \eta_{\mathrm{G}}^{2}=.002\right)$. High-frequency words elicited more positive-going ERP amplitudes than low-frequency words, and this difference was significant at $\mathrm{Pz}$ (high vs. low: $\mathrm{F}(1,37)=12.85, p=.001$, mean difference $=.40 \mu \mathrm{V}, 95 \% \mathrm{CI}$ for difference: .18 to .63 $\mu \mathrm{V}$ ) and $\mathrm{Cz}$ locations (high vs. low: $\mathrm{F}(1,37)=8.63$, $p=.006$, mean difference $=.34 \mu \mathrm{V}, 95 \% \mathrm{CI}$ for difference: $.10-.57 \mu \mathrm{V}$ ), but not at the $\mathrm{Fz}$ location (high vs. low: $\mathrm{F}(1,37)=.19, \quad p=.66 ; \quad$ mean difference $=-.05 \mu \mathrm{V}$, $95 \% \mathrm{CI}$ for difference: -.26 to $.17 \mu \mathrm{V})$. The main effect of Group did not reach significance $(\mathrm{F}(1,37)=1.02, p=.3)$
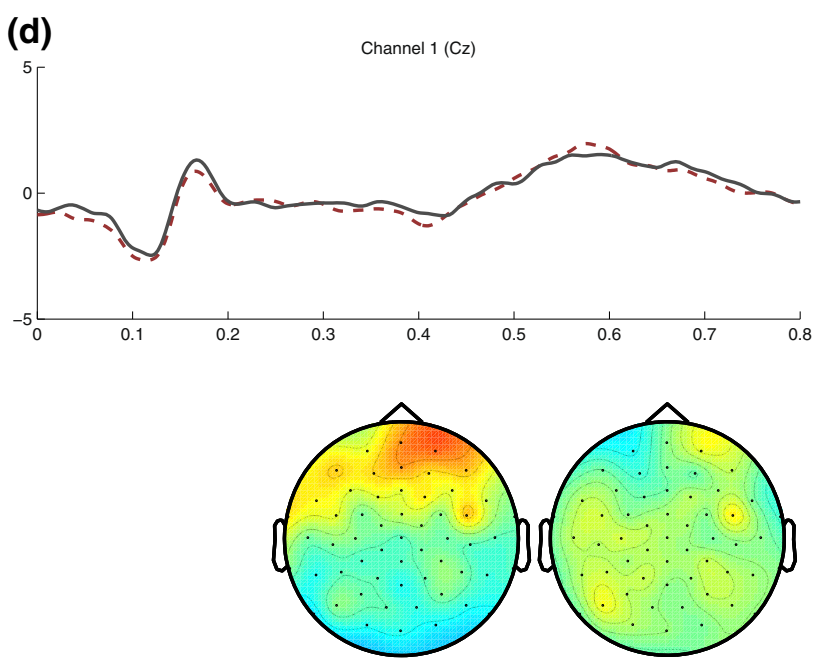

$300-500 \mathrm{~ms}$

$500-700 \mathrm{~ms}$

versus neutral words (gray line), c ASD group, positive words (green dashed line) versus neutral words (gray line), $\mathbf{d}$ ASD group, negative words (red dashed line) versus neutral words (gray line) (Color figure online)

and neither did any interactions involving Group (frequency by group: $\mathrm{F}(1,37)=1.6, p=.2$; Location by Group: $\mathrm{F}(2,74)=2.67, p=.1$; frequency by group by location: $\mathrm{F}(2,74)=.15, p=.8)$.

For the effect of valence, we ran a Group (ASD, typical) by Emotion (positive, negative, neutral) by Location $(\mathrm{Fz}$, $\mathrm{Cz}, \mathrm{Pz}$ ) repeated measures ANOVA with Group as between-subject factor, and Emotion and Location as within-subject factors. For the variables with more than three levels the $p$ values were adjusted with GreenhouseGeisser correction.

Within the N400 time window (300-500 ms), the ANOVA yielded a significant main effect of Emotion $\left(\mathrm{F}(2,74)=8.65, p<.001, \eta_{\mathrm{p}}^{2}=.19, \eta_{\mathrm{G}}^{2}=.003\right)$, main effect of Location $\left(\mathrm{F}(2,74)=28.32, p<.001, \eta_{\mathrm{p}}^{2}=.43\right.$, 
$\left.\eta_{\mathrm{G}}^{2}=.34\right)$, and a Group by Emotion interaction $\left(\mathrm{F}(2,74)=5.24, \quad p=.008, \quad \eta_{\mathrm{p}}^{2}=.12, \quad \eta_{\mathrm{G}}^{2}=.002\right)$. То resolve this interaction, we repeated the ANOVA in each group separately.

Within the typical group, the main effect of Emotion was significant $\left(\mathrm{F}(2,38)=11.33, p<.001, \eta_{\mathrm{p}}^{2}=.37\right.$, $\left.\eta_{\mathrm{G}}^{2}=.009\right)$. Neutral words elicited less positive-going ERP amplitudes than both emotionally negative words $\left(\mathrm{F}(1,19)=20.1, \quad p<.001, \eta_{\mathrm{p}}^{2}=.51, \eta_{\mathrm{G}}^{2}=.01 ;\right.$ mean difference (neg-neu) $=.49 \mu \mathrm{V}, 95 \% \mathrm{CI}$ for difference: $.26-.72 \mu \mathrm{V})$ and emotionally positive words $\left(\mathrm{F}(1,19)=12.7, \quad p=.002, \eta_{\mathrm{p}}^{2}=.4, \quad \eta_{\mathrm{G}}^{2}=.008 ;\right.$ mean difference (pos-neu) $=.40 \mu \mathrm{V}, 95 \% \mathrm{CI}$ for difference: $.16-.63 \mu \mathrm{V})$. Within the ASD group, we did not find a significant effect for either the main effect of Emotion $\left(\mathrm{F}(2,36)=1.68, p=.2, \eta_{\mathrm{p}}^{2}=.08\right)$, positive versus neutral $\left(\mathrm{F}(1,18)=3.82, \quad p=.07, \quad \eta_{\mathrm{p}}^{2}=.17\right.$, mean difference $.16 \mu \mathrm{V}, 95 \% \mathrm{CI}-.01-.34 \mu \mathrm{V})$; negative versus neutral $\left(\mathrm{F}(1,18)=.01, \quad p=.9, \quad \eta_{\mathrm{p}}^{2}=.001, \quad\right.$ mean difference $.01 \mu \mathrm{V}, 95 \% \mathrm{CI}-.22-.24 \mu \mathrm{V})$ or Emotion by Location interaction $\left(\mathrm{F}(2,36)=1.69, p=.19, \eta_{\mathrm{p}}^{2}=.09\right)$.

Within the LPC time window (500-700 ms), the ANOVA yielded following significant effects: main effect of Emotion $\left(\mathrm{F}(2,74)=4.75, \quad p=.01, \quad \eta_{\mathrm{p}}^{2}=.11\right.$, $\left.\eta_{\mathrm{G}}^{2}=.002\right)$, main effect of Location $(\mathrm{F}(2,74)=26.27$, $\left.p<.001, \eta_{\mathrm{p}}^{2}=.42, \eta_{\mathrm{G}}^{2}=.29\right)$, Emotion by Location interaction $\left(\mathrm{F}(4,148)=3.07, \quad p=.04, \quad \eta_{\mathrm{p}}^{2}=.08\right.$, $\left.\eta_{\mathrm{G}}^{2}=.003\right)$, and finally Group by Emotion interaction $\left(\mathrm{F}(2,74)=3.38, \quad p=.04, \quad \eta_{\mathrm{p}}^{2}=.08, \quad \eta_{\mathrm{G}}^{2}=.002\right)$. То resolve this interaction, we repeated the ANOVA in each group separately.

Within the typical group, the main effect of Emotion was significant $\left(\mathrm{F}(2,38)=7.76, \quad p=.002, \quad \eta_{\mathrm{p}}^{2}=.29\right.$, $\left.\eta_{\mathrm{G}}^{2}=.008\right)$. Emotionally negative words elicited more positive-going ERP compared to neutral words $\left(\mathrm{F}(1,19)=16.74, p=.001, \eta_{\mathrm{p}}^{2}=.47, \eta_{\mathrm{G}}^{2}=.01 ;\right.$ mean difference $=.42 \mu \mathrm{V}, 95 \% \mathrm{CI} .21-.64 \mu \mathrm{V}$ ) but emotionally positive words did not significantly differ from neutral $\left(\mathrm{F}(1,19)=2.15, \quad p=.13, \eta_{\mathrm{p}}^{2}=.12, \eta_{\mathrm{G}}^{2}=.003 ;\right.$ mean difference: $.19 \mu \mathrm{V}, 95 \% \mathrm{CI}-.06-.44 \mu \mathrm{V})$. Within the ASD group, we did not find a significant effect for either the main effect of Emotion $(\mathrm{F}(2,36)=.07, p=.9$, $\eta_{\mathrm{p}}^{2}=.004$; positive versus neutral: $\mathrm{F}(1,18)=.06, p=.81$, $\eta_{\mathrm{p}}^{2}=.003$, mean difference: $.03 \mu \mathrm{V}, 95 \% \mathrm{CI}-.20-$ $.26 \mu \mathrm{V}$; negative versus neutral: $\mathrm{F}(1,18)=.11, p=.74$, $\eta_{\mathrm{p}}^{2}=.006$, mean difference: $.04 \mu \mathrm{V}$, $95 \% \mathrm{CI}-.20-$ $.27 \mu \mathrm{V})$ or Emotion by Location interaction $(\mathrm{F}(2,36)=$ 2.23, $p=.11, \eta_{\mathrm{p}}^{2}=.11$ ).

To explore whether the group effect was co-determined by IQ, PPVT was added to the model. The Group by Emotion interaction remained significant (in the N400 time window: $\mathrm{F}(2,72)=5.11, p=.009, \eta_{\mathrm{p}}^{2}=.12$; in the LPC time window: $\left.\mathrm{F}(2,72)=3.39, p=.04, \eta_{\mathrm{p}}^{2}=.09\right)$, but the main effect of Emotion became nonsignificant (in the N400 time window: $\mathrm{F}(2,72)=.14, p=.87, \eta_{\mathrm{p}}^{2}=.004$; in the LPC time window: $\left.\mathrm{F}(2,72)=.56, p=.57, \eta_{\mathrm{p}}^{2}=.01\right)$. Furthermore, the PPVT by Location interaction was significant (in the $\mathrm{N} 400$ time window: $\mathrm{F}(1.27,45.7)=6.3$, $p=.011, \quad \eta_{\mathrm{p}}^{2}=.15 ; \quad$ in the LPC time window: $\left.\mathrm{F}(1.3,47.9)=10.44, p=.001, \eta_{\mathrm{p}}^{2}=.23\right)$. In participants with high PPVT scores, in both time windows the average voltage was more positive at posterior electrodes and more negative at frontal electrodes, but in participants with low PPVT scores the topography was more "flat", with a smaller difference between front and back. This was the case in both ASD and in typical group.

The whole surface analysis (which corrects for multiple comparisons and is unbiased with respect to selecting time windows and electrodes) did not find a significant EPN effect. To make sure that we did not miss the EPN, we tested for the effect of EPN in the time window and location reported by Schacht and Sommer 2009 (200-300 ms, electrodes 42 and 44 which correspond to $\mathrm{O} 1$ and $\mathrm{O} 2)$. We found no significant effect of emotion $(\mathrm{F}(2,74)=1.9, p=.16)$.

We also explored whether the ERP effect (defined as the voltage difference between emotion and neutral words at the $\mathrm{Cz}$ location in the 300-500 ms time window, where the LPC was largest) was correlated with the behavioral measure of interest (emotion facilitation effect). The correlation turned out to be not significant (for positive words: $\mathrm{r}=-.23, p=.17$; for negative words: $\mathrm{r}=.09, p=.6$ ).

\section{Discussion}

In the present lexical decision study, we compared the performance of high-functioning ASD participants and a typical comparison group on behavioral measures of reaction times and error rates, and on ERP amplitude in various time windows following stimulus onset. In line with previous research, the typical group gave faster and more accurate responses to emotion words compared to neutral words. Contrary to our prediction, we found a similar effect of emotional valence on the reaction times and error rates of the ASD group. This result shows that individuals with ASD are not "blind" or "insensitive" to valence, as we hypothesized.

Our behavioral findings contradict the initial hypothesis that subjects with ASD would process emotion and neutral words in a similar way, as well as previous studies finding no effect of emotion in the ASD group. There may be several explanations for the obtained result pattern. First, the task was different: Previous studies used memory and attention tasks requiring stimulus detection or recall, while our study involved a lexical decision task, which required 
giving a response on every trial. The two types of tasks tap into different stages of emotion processing: The emotional facilitation effect in the lexical decision is mainly driven by early preconscious bottom-up facilitation that speeds up recognition and response (Kousta et al. 2009), while successful emotional memory formation is associated with increased activity at a later time window during postlexical processing (Dolcos and Cabeza 2002).

Another explanation for the findings lies in the variation between participant samples. There is a possibility that other parameters, which we did not measure, such as callous-unemotional traits (Dolan and Fullam 2010; Rogers et al. 2006) or alexithymia (Bird et al. 2010, 2011; Lombardo et al. 2007), are responsible for differences between our sample and samples included in the other studies.

As a third option, we should consider that some of the previous studies that found the behavioral reactions to emotion words in ASD and typically developing participants to be different, based their conclusions on finding a significant effect in the typical group in the absence of an effect in the ASD group, while their main effects of group or the group by valence interaction did not reach significance (Gaigg and Bowler 2008, 2009a; Dichter et al. 2010). This throws some doubt on these studies with respect to their power to assess whether the ASD and control groups were really different or similar in behavioral performance.

With regard to ERP measures, we found an effect of lexicality (words vs. nonwords) and of word frequency (high vs. low) in both participant groups. The effect of frequency reached significance in the late N400 time window (400-500 ms) and had a similar spatial distribution in both groups. ROI analysis confirmed that in the N400 time window (300-500 ms), the ERP amplitude was significantly lower for low-frequency words in both groups with no between-group differences. This finding lends support to the validity of our experimental design and stimulus materials. As predicted, we found a significant effect of stimulus valence in the typical group, which consisted of a positive shift in the ERP at central and anterior electrodes at 350-400 ms, moving towards centroparietal electrodes at 500-600 ms. The distribution and timing of the effect closely resembled the LPC reported previously for emotional words and images (Dolcos and Cabeza 2002; Kissler and Koessler 2011). In the ASD group, the LPC effect was absent in both the positive versus neutral contrast and the negative vs neutral contrast. The group by valence interaction analyses confirmed that the negative versus neutral contrast, but not the positive versus neutral contrast, differed significantly by group. The same pattern emerged in the ROI analysis, where we found a significant group by valence interaction in both $\mathrm{N} 400$ and LPC time windows, with a significant effect of valence in the typical but not ASD group. We did not find a significant
EPN effect in either group; however, previous studies suggest that EPN is more dependent on task and stimuli characteristics (compared to LPC) and is more difficult to replicate (Dillon et al. 2006; Hofmann et al. 2009).

Based on the ERP data, we conclude that individuals with ASD may still process in particular negative emotional valence differently compared to typical participants, while the corresponding behavioral response data do not differ between the groups. The finding that the group by valence interaction only reached significance in the negative condition is consistent with the results of previous research (e.g., Deruelle et al. 2008), where a significant group by valence interaction emerged for negative, but not for positive stimuli. Further, studies of processing of emotion words and images suggest that negative valence is qualitatively different from positive valence, and has a stronger effect on brain activity and behavior (Dahl 2001; Nasrallah et al. 2009; Ohira et al. 1998; Vaish et al. 2008; Taylor 1991). In life, it is generally more important to avoid threatening objects than to approach attractive ones. Neural mechanisms affected in the ASD sample may be crucial for the processing of negative valence, but not so much positive valence, in line with the finding that the positive condition differed from neutral, albeit only at trend level, while the negative condition did not.

The absence of the LPC in the participants with ASD suggests that they do not engage in more intensive topdown processing during the comprehension of emotion words. It also suggests that they process valence in a way that differs from the typical population. In principle, the processes underlying the LPC should have no effect of the lexical decision, because the LPC occurs after lexical access. Thus, by the time the LPC reaches its peak (around 500-600 ms), the word has already been identified. On the other hand, LPC has been associated with increased cognitive processing load, and enhanced attention and memory encoding. In other studies, a larger ERP amplitude for emotion words in the 400-600 ms interval predicted better subsequent memory performance (Dolcos and Cabeza 2002). Therefore, the lack of ERP modulation by valence in the ASD group may explain why previous studies found no emotional memory effect in that group. Furthermore, the absence of an LPC in the ASD group is not likely to be due to general difficulty with language stimuli or lack of reliable ERP response to words. Note that the effect of valence in the typical group lasted even longer than the effect of frequency and also involved a larger cluster of electrodes. In contrast, in the ASD group, the effect of frequency was present, but there was no significant effect of valence. This indicates that it is not the processing of linguistic stimuli per se that makes the difference between ASD and typical participants, but their valence in particular. 
To summarize, our study led to a number of innovative findings and conclusions. Firstly, contrary to suggestions from earlier studies, individuals with ASD are not completely insensitive to emotional valence, as is reflected in our behavioral findings. However, and secondly, individuals with ASD process emotional valence in a different way than typical individuals on the neural level, as indicated by our EEG data. Whereas manipulations of lexicality and frequency evoked similar neural responses by the participants from both groups, the effect of valence (as reflected by LPC) was only present in the typical group.

The behavioral emotional facilitation is thought to be an early preconscious effect reflecting enhanced bottom-up processing (Kousta et al. 2009). On the other hand, LPC is generated by the cortical sources and is thought to reflect postlexical processing, allocation of additional resources for information that can be potentially relevant (Schupp et al. 2000, 2006). Our results suggest that the deficit in emotional processing in ASD is specific to late top-down processing, while the early stages of processing are unaffected. Perhaps the participants with ASD have developed an alternative processing strategy for emotional content which results in the atypical ERP response.

A limitation of this study is that ADI scores were available only for a subgroup of participants with ASD. Therefore, it might be argued that this limits a potential generalization of the findings to the ASD population as a whole. However, we would like to point out that the clinical diagnosis of ASD was beyond doubt in all participants with ASD.

In all, our results add to the understanding of emotion processing in high-functioning individuals with ASD. The existence and extent of emotional impairments in ASD has become a debated topic. Recent reviews indicate that individuals with ASD indeed have difficulty in processing emotional stimuli, but it is still debated whether those difficulties are secondary to other cognitive domains or constitute an independent problem (Nuske et al. 2013; Gaigg 2012). Our results support the theoretical position that emotional impairments extend beyond the visual domain to emotional language and are a relatively independent component of ASD. Further study of the neural architecture of abnormal emotion processing in ASD is warranted, and should be complemented with studies on how emotional impairments link with cognitive processes and social behavior in ASD. Already in 1943, Kanner described autistic children as emotionally cold and distanced. However, in the decades following this remark, this topic received relatively little attention, with most emotionrelated research in ASD focusing on the perception of emotions in faces. The present study meant to revitalize the important topic of the relations between emotion, language, and cognition. We hope to have shown that the study of emotion processing by means of linguistic stimuli may contribute significantly to our understanding of ASD.

Acknowledgments We are very grateful to all people with ASD who participated in this study. This work was supported by the Netherlands Organization for Scientific Research under the Top Talent Donders Graduate School grant 243301-24000246. Finally, we would like to acknowledge Dr Eelke Visser for his assistance.

\section{References}

APA. (2000). Diagnostic and statistical manual of mental disorders: DSM-IV-TR (4th text revision). Washington, DC: American Psychiatric Publishing Inc.

Baayen, R. H., Piepenbrock, R., \& Gulikers, L. (1995). The CELEX lexical database [CD-ROM]. University of Pennsylvania Linguistic Data Consortium.

Bakeman, R. (2005). Recommended effect size statistics for repeated measures designs. Behavior Research Methods, 37(3), 379-384.

Baron-Cohen, S., Wheelwright, S., Hill, J., Raste, Y., \& Plumb, I. (2001). The "reading the mind in the eyes" test revised version: A study with normal adults, and adults with asperger syndrome or high-functioning autism. Journal of Child Psychology and Psychiatry, 42(2), 241-251.

Bayer, M., Sommer, W., \& Schacht, A. (2010). Reading emotional words within sentences: The impact of arousal and valence on event-related potentials. International Journal of Psychophysiology, 78(3), 299-307.

Beversdorf, D. Q., Anderson, J. M., Manning, S. E., Anderson, S. L., Nordgren, R. E., Felopulos, G. J., et al. (1998). The effect of semantic and emotional context on written recall for verbal language in high functioning adults with autism spectrum disorder. Journal of Neurology, Neurosurgery and Psychiatry, 65, 685-692.

Bird, G., Press, C., \& Richardson, D. C. (2011). The role of alexithymia in reduced eye-fixation in autism spectrum conditions. Journal of Autism and Developmental Disorders, 41, 556-1564.

Bird, G., Silani, G., Brindley, R., White, S., Frith, U., \& Singer, T. (2010). Empathic brain responses in insula are modulated by levels of alexithymia but not autism. Brain, 133(5), $1515-1525$.

Chwilla, D. J., Kolk, H. H. J., \& Mulder, G. (2000). Mediated priming in the lexical decision task: Evidence from event-related potentials and reaction time. Journal of Memory and Language, 42(3), 314-341.

Corden, B., Chilvers, R., \& Skuse, D. (2008). Emotional modulation of perception in Asperger's syndrome. Journal of Autism and Developmental Disorders, 38, 1072-1080.

Dahl, M. (2001). Asymmetries in the processing of emotionally valenced words. Scandinavian Journal of Psychology, 42, 97-104.

Deruelle, C., Hubert, B., Santos, A., \& Wicker, B. (2008). Negative emotion does not enhance recall skills in adults with autistic spectrum disorders. Autism Research, 1, 91-96.

Dichter, G. S., Benning, S. D., Holtzclaw, T. N., \& Bodfish, J. W. (2010). Affective modulation of the startle eyeblink and postauricular reflexes in autism spectrum disorder. Journal of Autism and Developmental Disorders, 40, 858-869.

Dillon, D. G., Cooper, J. J., Grent-'t-Jong, T., Woldorff, M. G., \& LaBar, K. S. (2006). Dissociation of event-related potentials indexing arousal and semantic cohesion during emotional word encoding. Brain and Cognition, 62(1), 43-57. 
Dolan, M. C., \& Fullam, R. (2010). Emotional memory and psychopathic traits in conduct disordered adolescents. Personality and Individual Differences, 48(3), 327-331.

Dolcos, F., \& Cabeza, R. (2002). Event-related potentials of emotional memory: Encoding pleasant, unpleasant, and neutral pictures. Cognitive, Affective, \& Behavioral Neuroscience, 2(3), 252-263.

Eviatar, Z., \& Zaidel, E. (1991). The effects of word length and emotionality on hemispheric contribution to lexical decision. Neuropsychologia, 29(5), 415-428.

Gaigg, S. B. (2012). The interplay between emotion and cognition in autism spectrum disorder: Implications for developmental theory. Frontiers in Integrative Neuroscience, 6, 113. doi:10.3389/ fnint.2012.00113.

Gaigg, S. B., \& Bowler, D. M. (2008). Free recall and forgetting of emotionally arousing words in autism spectrum disorder. Neuropsychologia, 46, 2336-2343.

Gaigg, S. B., \& Bowler, D. M. (2009a). Brief report: Attenuated emotional suppression of the attentional blink in autism spectrum disorder: Another non-social abnormality? Journal of Autism and Developmental Disorders, 39, 1211-1217.

Gaigg, S. B., \& Bowler, D. M. (2009b). Illusory memories of emotionally charged words in autism spectrum disorder: Further evidence for atypical emotion processing outside the social domain. Journal of Autism and Developmental Disorders, 39(7), 1031-1038.

Grainger, J. (1990). Word frequency and neighborhood frequency effects in lexical decision and naming. Journal of Memory and Language, 29(2), 228-244.

Groen, W. B., Orsouw, L., Huurne, N., Swinkels, S., Gaag, R.-J., Buitelaar, J., et al. (2009). Intact spectral but abnormal temporal processing of auditory stimuli in autism. Journal of Autism and Developmental Disorders, 39(5), 742-750.

Halgren, E., Dhond, R. P., Christensen, N., Van Petten, C., Marinkovic, K., Lewine, J. D., et al. (2002). N400-like magnetoencephalography responses modulated by semantic context, word frequency, and lexical class in sentences. NeuroImage, 17(3), 1101-1116.

Harms, M. B., Martin, A., \& Wallace, G. L. (2010). Facial emotion recognition in autism spectrum disorders: A review of behavioral and neuroimaging studies. Neuropsychology Review, 20, 290-322.

Hauk, O., \& Pulvermüller, F. (2004). Effects of word length and frequency on the human event-related potential. Clinical Neurophysiology, 115(5), 1090-1103.

Heerey, E. A., Keltner, D., \& Capps, L. M. (2003). Making sense of self-conscious emotion: Linking theory of mind and emotion in children with autism. Emotion, 3(4), 394-400.

Herbert, C., Junghofer, M., \& Kissler, J. (2008). Event related potentials to emotional adjectives during reading. Psychophysiology, 45(3), 487-498.

Herbert, C., Kissler, J., Junghöfer, M., Peyk, P., \& Rockstroh, B. (2006). Processing of emotional adjectives: Evidence from startle EMG and ERPs. Psychophysiology, 43(2), 197-206.

Hillier, A., \& Allinson, L. (2002). Beyond expectations: Autism, understanding embarrassment, and the relationship with theory of mind. Autism, 6(3), 299-314.

Hofmann, M. J., Kuchinke, L., Tamm, S., Võ, M. L. H., \& Jacobs, A. M. (2009). Affective processing within 1/10th of a second: High arousal is necessary for early facilitative processing of negative but not positive words. Cognitive, Affective, \& Behavioral Neuroscience, 9(4), 389-397.

Holcomb, P. J., \& Grainger, J. (2006). On the time-course of visual word recognition: En ERP investigation using masked repetition priming. Journal of Cognitive Neuroscience, 18(10), 1631-1643.
Holt, D. J., Lynn, S. K., \& Kuperberg, G. R. (2008). Neurophysiological correlates of comprehending emotional meaning in context. Journal of Cognitive Neuroscience, 21(11), 2245-2262.

Jemel, B., Mottron, L., \& Dawson, M. (2006). Impaired face processing in autism: Fact or artifact? Journal of Autism and Developmental Disorders, 36(1), 91-106.

Kanske, P., \& Kotz, S. A. (2007). Concreteness in emotional words: ERP evidence from a hemifield study. Brain Research, 1148, $138-148$.

Kensinger, E., \& Corkin, S. (2003). Memory enhancement for emotional words: Are emotional words more vividly remembered than neutral words? Memory \& Cognition, 31(8), 1169-1180.

Kilner, J. M. (2013). Bias in a common EEG and MEG statistical analysis and how to avoid it. Clinical Neurophysiology, 124(10), 2062-2063.

Kissler, J., Herbert, C., Winkler, I., \& Junghofer, M. (2009). Emotion and attention in visual word processing-an ERP study. Biological Psychology, 80(1), 75-83.

Kissler, J., \& Koessler, S. (2011). Emotionally positive stimuli facilitate lexical decisions-an ERP study. Biological Psychology, 86(3), 254-264.

Kousta, S.-T., Vinson, D. P., \& Vigliocco, G. (2009). Emotion words, regardless of polarity, have a processing advantage over neutral words. Cognition, 112(3), 473-481.

Kuchinke, L., Võ, M. L. H., Hofmann, M., \& Jacobs, A. M. (2007). Pupillary responses during lexical decisions vary with word frequency but not emotional valence. International Journal of Psychophysiology, 65(2), 132-140.

Lombardo, M. V., Barnes, J. L., Wheelwright, S. J., \& Baron-Cohen, S. (2007). Self-referential cognition and empathy in autism. PLOS ONE, 2(9), e883.

Lord, C., Rutter, M., \& Couteur, A. (1994). Autism diagnostic interview-revised: A revised version of a diagnostic interview for caregivers of individuals with possible pervasive developmental disorders. Journal of Autism and Developmental Disorders, 24(5), 659-685.

Mackay, D. G., Shafto, M., Taylor, J. K., Marian, D. E., Abrams, L., \& Dyer, J. R. (2004). Relations between emotion, memory, and attention: Evidence from taboo Stroop, lexical decision, and immediate memory tasks. Memory \& Cognition, 32(3), 474-488.

Manschot, W., \& Bonnema, J. T. (1974). Handleiding bij de experimentele Nederlandse normering van de Peabody Picture Vocabulary Test [Manual of the experimental Dutch norms of the Peabody Picture Vocabulary Test]. Lisse: Swets \& Zeitlinger.

Maris, E., \& Oostenveld, R. (2007). Nonparametric statistical testing of EEG- and MEG-data. Journal of Neuroscience Methods, 164(1), 177-190.

Moratti, S., Saugar, C., \& Strange, B. A. (2011). Prefrontaloccipitoparietal coupling underlies late latency human neuronal responses to emotion. The Journal of Neuroscience, 31(47), 17278-17286.

Nasrallah, M., Carmel, D., \& Lavie, N. (2009). Murder, she wrote: Enhanced sensitivity to negative word valence. Emotion, 9(5), 609-618.

Nuske, H. J., Vivanti, G., \& Dissanayake, C. (2013). Are emotion impairments unique to, universal, or specific in autism spectrum disorder? A comprehensive review. Cognition \& Emotion, 27(6), 1042-1061.

Ohira, H., Winton, W. M., \& Oyama, M. (1998). Effects of stimulus valence on recognition memory and endogenous eyeblinks: Further evidence for positive-negative asymmetry. Personality and Social Psychology Bulletin, 24(9), 986-993.

Oostenveld, R., Fries, P., Maris, E., \& Schoffelen, J.-M. (2011). FieldTrip: Open source software for advanced analysis of MEG, 
EEG, and invasive electrophysiological data. Computational Intelligence and Neuroscience, 2011. doi:10.1155/2011/156869.

Poljac, E., Simon, S., Ringlever, L., Kalcik, D., Groen, W. B., Buitelaar, J. K., et al. (2009). Impaired task switching performance in children with dyslexia but not in children with autism. The Quarterly Journal of Experimental Psychology, 63(2), 401-416.

Raven, J., Raven, J. C., \& Court, J. H. (1998). Raven manual section 4: Advanced progressive matrices. Oxford: Oxford Psychologists Press.

Rogers, J., Viding, E., Blair, J. R., Frith, U., \& Happe, F. (2006). Autism spectrum disorder and psychopathy: Shared cognitive underpinnings or double hit? Psychological Medicine, 36(12), 1789-1798.

Rugg, M. (1990). Event-related brain potentials dissociate repetition effects of high-and low-frequency words. Memory \& Cognition, 18(4), 367-379.

Schacht, A., \& Sommer, W. (2009). Time course and task dependence of emotion effects in word processing. Cognitive, Affective, \& Behavioral Neuroscience, 9(1), 28-43.

Schupp, H. T., Cuthbert, B. N., Bradley, M. M., Cacioppo, J. T., Ito, T., \& Lang, P. J. (2000). Affective picture processing: The late positive potential is modulated by motivational relevance. Psychophysiology, 37(2), 257-261.

Schupp, H. T., Flaisch, T., Stockburger, J., \& Junghöfer, M. (2006). Emotion and attention: Event-related brain potential studies. In G. E. M. J. J. K. S. Anders, \& D. Wildgruber (Eds.), Progress in brain research, Vol. 156, (pp. 31-51). Amsterdam: Elsevier.

South, M., Ozonoff, S., Suchy, Y., Kesner, R. P., McMahon, W. M., \& Lainhart, J. E. (2008). Intact emotion facilitation for nonsocial stimuli in autism: Is amygdala impairment in autism specific for social information? Journal of the International Neuropsychological Society, 14, 42-54.
Taylor, S. E. (1991). Asymmetrical effects of positive and negative events: The mobilization-minimization hypothesis. Psychological Bulletin, 110(1), 67-85.

Vaish, A., Grossmann, T., \& Woodward, A. (2008). Not all emotions are created equal: The negativity bias in social-emotional development. Psychological Bulletin, 134(3), 383-403.

van Ede, F., de Lange, F., Jensen, O., \& Maris, E. (2011). Orienting attention to an upcoming tactile event involves a spatially and temporally specific modulation of sensorimotor alpha- and betaband oscillations. The Journal of Neuroscience, 31(6), 2016-2024.

van Hell, J. G., \& Dijkstra, T. (2002). Foreign language knowledge can influence native language performance in exclusively native contexts. Psychonomic Bulletin \& Review, 9(4), 780-789.

Visser, E., Zwiers, M. P., Kan, C. C., Hoekstra, L., van Opstal, A. J., \& Buitelaar, J. K. (2013). Atypical vertical sound localization and sound-onset sensitivity in autism spectrum disorders. Journal of Psychiatry and Neuroscience, 38(6), 398-406.

Wald, F. D., \& Mellenbergh, G. J. (1990). De verkorte versie van de Nederlandse vertaling van de Profile of Mood States (POMS). [The shortened version of the Dutch translation of the Profile of Mood States (POMS).]. Nederlands Tijdschrift voor de Psychologie en haar Grensgebieden, 45(2), 86-90.

Wilbarger, J. L., McIntosh, D. N., \& Winkielman, P. (2009). Startle modulation in autism: Positive affective stimuli enhance startle response. Neuropsychologia, 47, 1323-1331.

Yap, M. J., Balota, D. A., \& Tan, S. E. (2013). Additive and interactive effects in semantic priming: Isolating lexical and decision processes in the lexical decision task. Journal of Experimental Psychology: Learning, Memory, and Cognition, 39(1), 140-158. doi:10.1037/a0028520. 